\title{
EFFECT OF AMINO ACIDS AS GROWTH STIMULANT FOR RECOVERY OF SHIGELLA SPP.
}

\author{
Ashfaqul Alam, Lutfe Ara, Anowara Begum* and Sirajul Islam Khan \\ Department of Microbiology, University of Dhaka, Dhaka-1000, Bangladesh
}

Key words: Response, Amino acid, Shigella spp., Enrichment

Shigellosis is the most virulent and serious, due to its invasive character, systemic manifestations, severe nutritional impact and tendency to become recurrent over prolonged period.(1) In Bangladesh, among the routine hospitalized diarrhoeal patients, the isolation rate of shigellae are approximately $12 \%$.(2) S. flexneri and S. dysenteriae type 1 are the two predominant serotypes isolated in Bangladesh as also in other developing countries. S. flexneri alone constituted about $60 \%$ of the shigellosis (ICDDR,B 1980), the number described to $42 \%$ in 1984.(3) The presence of Shigella is almost never been sought in routine water testing. ${ }^{(4)}$ This might be due to several factors including stress or injury of cells, viable but non-culturable state, or constrains of routine biological media for the isolation of Shigella.(5) In the aquatic environment, adverse environmental condition e.g., starvation or exposure to sublethal temperature, salinity or toxic chemicals may stress bacteria, which are capable of growth in warm blooded animal. Because of such sublethal injury or stress, bacteria may enter a viable but non-culturable condition, which has been defined by Nilson(6) as an inability of cells to produce colonies on solid media even following prolonged incubation. Shigella spp. have also been found to enter such viable but non-culturable state in laboratory condition. Recently Colwell et al.(7) has shown that viable but non-culturable cells of $S$. dysenteriae type 1 retained several virulence factors and remained potentially virulent, possing a public health problem. Thus, the potential public health hazard initiated by Shigella species existing in non-culturable state may be significant. Conventional bacteriological culture media/methods are inadequate to detect such cells and special resuscitation methods are required ${ }^{6}$. The cells might re-grow when the environmental conditions become favourable for growth. ${ }^{(8)}$ This study envisages to assess the influence of certain essential amino acids as growth stimulants leading to the recovery of stressed Shigella spp. in conventional culture media following newly designed pre-enrichment and enrichment media.

The following amino acids were examined as growth stimulants e.g., DL-alanine, Lalanine, L-arginine, L-aspartic acid, L-cystein, L-glutamic acid, glycine, L-histidine, Llysine, L-tryptophane and L-tyrosine. The chemically defined basal medium (CDM) was designed to culture and recovery of Shigella spp. in this study. The compositions of CDM

*Corresponding author. <anowara71@yahoo.com>. 
were (g/l): mannitol, 5.0; glucose, 2.0; $\mathrm{NH}_{4} \mathrm{Cl}, 0.2 ; \mathrm{K}_{2} \mathrm{HPO}_{4}$, 2.0; $\mathrm{KH}_{2} \mathrm{PO}_{4}, 1.0 ; \mathrm{MgSO}_{4} .7 \mathrm{H}_{2} \mathrm{O}$, $0.2 ; \mathrm{CaCl}_{2} .2 \mathrm{H}_{2} \mathrm{O}, 0.1$ and $\mathrm{MnSO}_{4} . \mathrm{H}_{2} \mathrm{O}, 10.0 \mathrm{mg}$; Ferric citrate, $5.0 \mathrm{mg} ; \mathrm{ZnSO}_{4} .7 \mathrm{H}_{2} \mathrm{O}, 2.0 \mathrm{mg}$; $\mathrm{CuSO}_{4}, 1.0 \mathrm{mg}$; Na-molybdate, $0.5 \mathrm{mg}$; agar $20.0 \mathrm{~g}$ and water 1.0 lit. After sterilization of the above mentioned ingredients for CDM, the filter sterilized amino acid $(0.001 \mathrm{~g} / \mathrm{ml})$ was added. In this study, S. dysenteriae type 1 (strain nos. 27914 and 9860) and S. flexneri (strain nos. 27450 and 27682) were used. The survival pattern of Shigella spp. was carried out in $99 \mathrm{ml}$ sterile phosphate buffer inoculated with $1 \mathrm{ml}$ of bacterial suspension, which resulted in the final concentration of approximately $2.76 \times 10^{5}$ cells $/ \mathrm{ml}$. The flasks were then incubated at room temperature $\left(25^{\circ} \mathrm{C}\right)$. All counts were done on nutrient agar, XLD, MacConkey's agar as standard and the CDM. The number of Shigella cells were decreased from day 1 to day 5 gradually and recovery rate on nutrient agar, XLD, MacConkey and CDM were almost same because here we used the known inoculum of Shigella cells for survival study. After 5 days, the cells of Shigella spp. decreased drastically and became non-culturable at 9th day and did not grow on NA, XLD, MacConkey agar media except CDM containing different amino acids. So, we concluded that amino acids had some influence for resuscitation of viable but non-culturable cells of Shigella spp.

Culturable cells were counted at $0,1,2,4,8$ and 24 hrs and then at consecutive days until the bacteria were no longer culturable. Two days after the apparent "die-off", $5.0 \mathrm{ml}$ of sample was removed from each of the microcosm and transferred to $45 \mathrm{ml}$ of having decreasing strength e.g., $1 / 2,1 / 5,1 / 10,1 / 50$ and $1 / 100$ of the pre-enrichment media. The composition of pre-enrichment media was (g/l): glucose 5.0; peptone 5.0; yeast extract 5.0; $\mathrm{K}_{2} \mathrm{HPO}_{4}$ 2.0; $\mathrm{KH}_{2} \mathrm{PO}_{4} 1.0 ; \mathrm{NaCl} 1.0 ;$ Na-pyruvate 0.50; Na-glutamate 0.50; arginine 0.25; trytophane 0.50 and distilled water $1.0 \mathrm{l}$. Plate counting was performed at every $6 \mathrm{hrs}$ interval according to drop plate method using nutrient agar, XLD, MacConkey and CDM.

The newly formulated chemically defined medium supported excellent growth of $S$. dysenteriae and S. flexneri and was to response to various amino acids. Both the strain of $S$. dysenteriae and S. flexneri were found to preferentially utilize L-glutamic acid. L-arginine was significantly used by $S$. dysenteriae but $S$. flexneri showed good growth while in case of L-aspartic acid, they showed opposite response. The growth of S. flexneri was good with L-alanine and L-cysteine but response was poor with $S$. dysenteriae. No significant growth response was observed with DL-alanine, glycine, L-histidine, L-lysine, L-tyrosine, only S. flexneri 27450 showed good growth in presence of lysine. Both the strain of Shigella spp. responded well with tryptophane. Response of Shigella spp. to different amino acids has been described in Table 1 .

Viable cell number of Shigella decreased sharply in phosphate buffered saline indicating a stressed condition due to lack of nutrients. The initial count of $5.54 \times 10^{5} / \mathrm{ml}$ decreased after nine days there appeared no culturable cells in the microcosm (data not 
shown). Nutrient stressed cells of Shigella spp. were successfully recovered by the newly designed enrichment media from its non-culturable stage indicating apparent resuscitation. Within $10-12 \mathrm{hrs}$ viable count of Shigella reached up to a number of about $7.95 \times 10^{7} \mathrm{cfu} / \mathrm{ml}$.

Table 1. Response of Shigella spp. to certain essential amino acids in the chemically defined medium (CDM).

\begin{tabular}{lcccccccccccc}
\hline & \multicolumn{11}{c}{ Amino acids } \\
\cline { 2 - 11 } Shigella spp. & 1 & 2 & 3 & 4 & 5 & 6 & 7 & 8 & 9 & 10 & 11 \\
\hline S. dysenteriae type1 (9860) & $(+)$ & + & +++ & ++ & $(+)$ & +++ & $(+)$ & - & + & ++ & + \\
S. dysenteriae type1 (27914) & $(+)$ & + & +++ & ++ & $(+)$ & +++ & $(+)$ & $(+)$ & $(+)$ & ++ & $(+)$ \\
S. flexneri (27682) & + & ++ & ++ & +++ & ++ & +++ & + & + & + & ++ & $(+)$ \\
S. flexneri (27450) & + & ++ & ++ & +++ & ++ & +++ & + & + & ++ & ++ & + \\
\hline
\end{tabular}

1 = DL-alanine, 2 = L-alanine, 3 = L-arginine, 4 = L-aspartic acid, 5 - L-cysteine, 6 = L-glutamic acid,

7 = glycine, 8 = L-histidine, $9=$ L-lysine, $10=$ L-tryptophane, $11=\mathrm{L}$ - tyrosine.

- = No growth, $(+)=$ Fair growth, $+=$ Reasonably good growth, $++=$ Good growth,$+++=$ Excellent growth .

With the aim to formulate the pre-enrichment and enrichment culture media that can recover Shigella cells from environmental samples, the response of 11 amino acids was examined in the newly designed chemically defined medium. Complex media e.g. nutrient agar or plate count agar (PCA) were not used because these contain various organic nutrients, growth factors, vitamins and amino acids etc., those are already present in the media which may mask or produce artifact in the response study. $S$. dysenteriae was found preferentially to utilize L-glutamic acid and L-arginine followed by L-aspartic acid and L-alanine. The response of S. flexneri to the addition of L-glutamic acid and L-aspartic acid was significant. The relatively less growth response was noted with L-arginine and L-alanine. It is evident from this study that these amino acids are essential for the growth of $S$. dysenteriae and S. flexneri but probably they themselves cannot synthesize them. So, if these amino acids are supplemented in the growth medium, which might be repaired the stressed Shigella and enables their recovery on isolation media following enrichment. The addition of amino acids that caused positive response might be useful in the formulating new media that may enrich or isolate stressed or injured cells ${ }^{5}$. The amino acids which showed minimal response may not be essential to incorporate into the media to recover the injured cells.

In this study, attempts were made to stimulate the nutrient stressed conditions. Viable count of S. flexneri and S. dysenteriae type1 cells in the phosphate buffered saline (PBS) in microcosms decreased sharply indicating a stressed condition due to lack of growth promoting nutrients. 


\section{References}

1. Khan MU and G Curlin 1974. Shigella dysentery: a new health hazard in Bangladesh. Bangladesh Med. J. 3: 42-46.

2. Khan MU, G Curlin and MI Huq 1979. Epidemiology of Shigella dysenteriae type 1 infections in Dacca urban area. Trop. Geogr. Med. 31: 213-223.

3. Khan MU and M Shahidullah 1980. Contrasting epidemiology of Shigella dysenteriae and Shigella flexneri, Dacca. Trans. U. Soc. Trop. Med. Hyg. 74(4): 528-533.

4. Hendricks CW 1972. Enteric bacterial growth rates in river water. Appl. Microbiol. 24: 168-174.

5. Islam MS, MK Hasan, MA Miah, GC Sur, A Felsenstein, M Venkatesan, RB Sack and MJ Albert 1993. Use of polymerase chain reaction and fluorescent antibody methods for detecting viable but nonculturable Shigella dysenteriae type 1 in laboratory microcosms. Appl. Environ. Microbiol. 59(2): 536-540.

6. Nilsson L, JD Oliver and S Kjelberg 1991. Resuscitation and virulence towards mice of viable but nonculturable Vibrio vulnificus. Appl. Environ. Microbiol. 61(7): 2620-2630.

7. Colwell RR, ML Tamplin, PR Brayton, AL Tavgens, DB Hall, D Herrington, S Hall, A Huq and DA Sack 1990. Environmental aspects of Vibrio cholerae in transmission of cholera, p. 327343. In: RB Sack and R Zinnaki (ed). Advances in research on cholera and related areas. $7^{\text {th }}$ ed. KTK Scientific Publishers, Tokyo, Japan.

8. Dukan S, Y Levi and D Touati 1997. Recovery of culturability of an HOCL-stressed population of Escherichia coli after incubation in phosphate buffer: resuscitation or regrowth? Appl. Environ. Microbiol. 63: 4204-4209.

(Manuscript received on 24 April, 2010; revised on 18 July, 2010) 